\title{
A Miniature Fiber Tip Polystyrene Microsphere Temperature Sensor With High Sensitivity
}

\author{
Haibin $\mathrm{CHEN}^{1,2}$, Tianchong XIE ${ }^{1}$, Jiashuang $\mathrm{FENG}^{1}$, Xiongxing ZHANG ${ }^{1,2 *}$, \\ Wei WANG ${ }^{1,2}$, Yanping $\mathrm{LI}^{1}$, and Zilong GUO ${ }^{1,2}$ \\ ${ }^{1}$ School of Optoelectronic Engineering, Xi'an Technological University, Xi'an 710021, China \\ ${ }^{2}$ Shaanxi Province Key Lab of Photoelectric Measurement and Instrument Technology, Xi'an Technological University, \\ Xi'an 710021, China \\ ${ }^{*}$ Corresponding author: Xiongxing ZHANG \\ E-mail: zhangxiongxing@xatu.edu.cn
}

\begin{abstract}
A fiber-optic temperature sensor based on fiber tip polystyrene microsphere is proposed. The sensor structure can be formed simply by placing and fixing a polystyrene microsphere on the center of an optical fiber tip. Since polystyrene has a much larger thermal expansivity, the structure can be used for high-sensitive temperature measurement. By the illuminating of the sensor with a broadband light source and through the optical Fabry-Perot interference between the front and back surfaces of the polystyrene microsphere, the optical phase difference (OPD) or wavelength shift can be used for the extraction of temperature. Temperature measurement experiment shows that, using a fiber probe polystyrene microsphere temperature sensor with a spherical diameter of about $91.7 \mu \mathrm{m}$, a high OPD-temperature sensitivity of about $-0.61796 \mathrm{~nm} /{ }^{\circ} \mathrm{C}$ and a good linearity of 0.9916 were achieved in a temperature range of $20{ }^{\circ} \mathrm{C}-70{ }^{\circ} \mathrm{C}$.
\end{abstract}

Keywords: Polystyrene microsphere; fiber-optic sensor; Fabry-Perot interference; temperature measurement

Citation: Haibin CHEN, Tianchong XIE Jiashuang FENG, Xiongxing ZHANG, Wei WANG, Yanping LI, et al., "A Miniature Fiber Tip Polystyrene Microsphere Temperature Sensor With High Sensitivity,” Photonic Sensors, 2022, 12(1): 84-90.

\section{Introduction}

Fiber-optic Fabry-Perot (FP) sensors, as one important type of fiber-optic sensors, for their advantages of compact size, light weight, high sensitivity, strong anti-electromagnetic interference, etc, can be used in many important areas such as civil engineering, petroleum, aviation, biology, and medicine [1-5].

Particularly, the fiber-tip FP sensors, by the forming of the FP cavities on the fiber facets, due to the features of simpler structure, more compact size, and versatile to be used, have attracted much more attentions. The fiber tip FP sensors can be formed by attaching a dielectric, metal or polymer film on the fiber facet [6-9], fabricating an FP structure through micro electro mechanical system (MEMS) processing and then attaching it on a fiber facet $[10$, 11], the writing of a micro FP cavity near the fiber endface through the femtosecond laser micromachining technology $[12,13]$, and many other methods. These fiber tip FP sensors have been proposed for the sensing of various physical, chemical, or biological parameters such as temperature, pressure, $\mathrm{pH}$ value, and chemical concentration.

Recently, there appeared some proposals on the fabrication of the fiber tip FP sensors through the

Received: 21 August 2020 / Revised: 04 December 2020

(C) The Author(s) 2021. This article is published with open access at Springerlink.com

DOI: $10.1007 / \mathrm{s} 13320-021-0625-6$

Article type: Regular 
introducing of air bubbles or microspheres on the fiber facets [14-20]. Ma et al. [16] proposed the fabrication of a fiber-tip microcavity sensor by fusing a silica capillary to the end of a single mode fiber (SMF), tapering the capillary with internal gas pressure applied, and then melting the capillary at the waist to form an air-cavity. The cavity length of the sensor was only about $107 \mu \mathrm{m}$, and can be used for the measurement of pressure up to $40 \mathrm{MPa}$ [16]. Chen et al. [17] reported a fiber tip FP interferometric sensor made by the gluing of a glass microsphere at the etched end of a multimode optical fiber. However, the sensor showed a large nonlinearity, and the temperature sensitivity was about $0.2026 \mathrm{~nm} /{ }^{\circ} \mathrm{C}$ [17]. Jiang et al. [18] fabricated a microcavity on the tip of a multimode fiber through the arc discharge on a multimode fiber which was firstly made a hole on the facet by a chemical etching method, showing a good temperature linearity in a temperature range of $20^{\circ} \mathrm{C}$ to $1000{ }^{\circ} \mathrm{C}$ and a wavelength-temperature sensitivity of $0.00189 \mathrm{~nm} /{ }^{\circ} \mathrm{C}$ [18]. The fiber-tip temperature sensors proposed above showed relatively high requirements on the fabrication process. And since the material composed of the FP structure was silica, which has a relatively low thermal expansion coefficient, their sensitivities were relatively low.

There has been a standard way to mass fabricate monodisperse polymer microspheres through the dispersion polymerization method [21]. If these microspheres can be assembled onto the fiber tip, the fabrication process can be simplified effectively. Furthermore, since polymers commonly show a relatively large thermal expansion coefficient, the sensitivity can also be greatly improved.

In this paper, we demonstrate a novel miniature fiber tip temperature sensor which is made by the fixing of a polystyrene microsphere in the centre of a vertically cut single mode fiber (SMF). Using a wideband light source to illuminate the sensor, through the FP interference between the back and front surfaces of the microsphere, the optical phase difference (OPD) variation or the wavelength shift directly can be used for the extraction of environment temperature. The proposed sensor structure shows advantages of compact size, simple fabrication process, and high temperature sensitivity.

\section{Structure and fabrication}

The sensor structure is very simple, as shown in Fig. 1, which is only composed of a vertically cut SMF, a polystyrene microsphere attached to the center of the fiber tip, and a short glass capillary used for the mechanical protection.

As seen in Fig. 2, the fabrication procedures of the sensor are as follows:

Step 1: Remove a short length of the SMF coating layer near the fiber tip, and cut the fiber vertically to form a flat facet, as shown in Fig. 2(a).

Step 2: Cut a short-length glass capillary with inner diameters slightly larger than the outer diameter of the SMF, and penetrate the SMF into the glass capillary, as s shown in Fig. 2(b).

Step 3: Glue the polystyrene microsphere on the center of the fiber tip: spread a few of ultraviolet (UV)-curable epoxy on the fiber tip, as s shown in Fig. 2(c). Use a hollow-core fiber with an inner diameter of $75 \mu \mathrm{m}$, which is fixed on a multi-axis translation stage, to adsorb a polystyrene microsphere onto its end through the capillary effect. Use the multi-axis translation stage to move the polystyrene microsphere to the center of the fiber tip. Since the UV-curable epoxy has a larger surface tension than the solvent of the microsphere, after the attaching of the microsphere and the epoxy on the fiber tip, the microsphere will be attracted to the fiber tip. Move back to the translation stage, the microsphere will be left on the fiber tip. Using a UV light-emitting diode (LED) to illuminate the structure, the microsphere will be fixed in the center of the fiber tip, as seen in Fig. 2(d).

Step 4: Fix the glass capillary. Move back the fiber with microsphere till the microsphere is completely contained in the glass capillary, as seen in Fig.2(e), then spread a few UV-curable epoxy at the rear end (the end far away from the polystyrene microsphere) of the glass capillary, and illuminate it 
by the UV LED to fix the glass capillary on the SMF, as seen in Fig. 2(f).

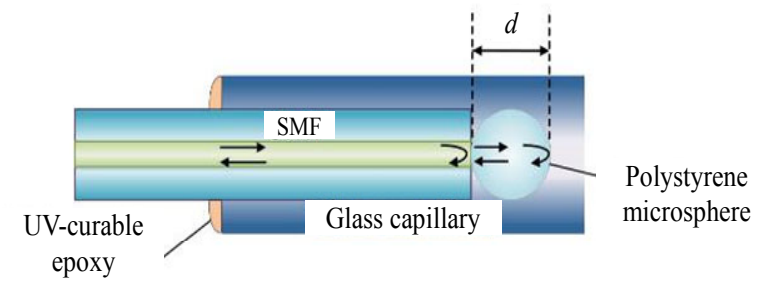

Fig. 1 Schematic diagram of the fiber tip polystyrene microsphere temperature sensor.

(a)

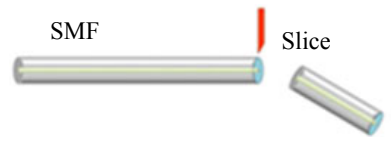

(b)

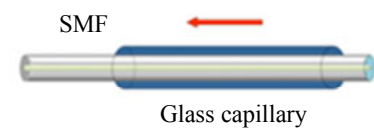

(c)

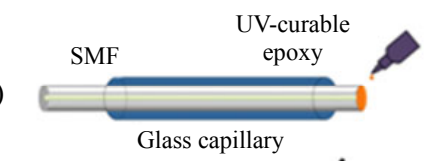

(d)

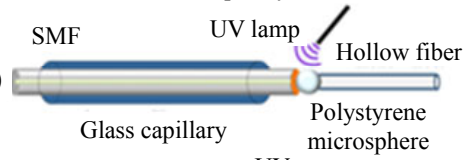

(e)

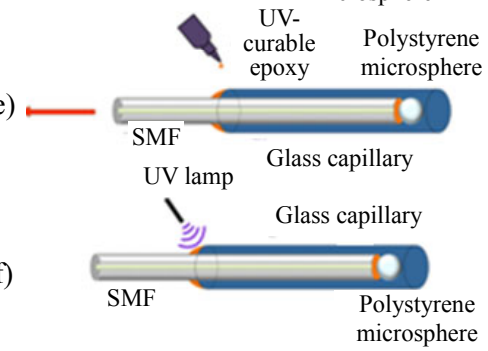

Fig. 2 Fabrication process of the fiber tip polystyrene microsphere sensor: (a) SMF cutting, (b) glass capillary penetration, (c) UV adhesive dispense, (d) polystyrene microsphere gluing, (e) glass capillary gluing, and (f) UV adhesive solidification.

The microspheres used were monodispersed polystyrene microspheres fabricated by Tianjin BaseLine ChromTech Research Centre (UniBead), which had an average diameter of $100 \mu \mathrm{m}$, the standard deviation was estimated to be $5 \%-10 \%$. The monodispersed polystyrene microspheres were suspended in a mixture solvent of 1:1 deionized water-ethanol $(2.5 \% \mathrm{w} / \mathrm{v})$. Figure 3(a) shows a microscopic photograph of the microspheres, in which the molecular formula was also given. The refractive index of the microspheres was 1.57. For a temperature below $80{ }^{\circ} \mathrm{C}$, polystyrene microspheres shrunk with the temperature increasing, since it has a negative thermal expansion coefficient. When the temperature was above the glass transition temperature $\left(80{ }^{\circ} \mathrm{C}-100{ }^{\circ} \mathrm{C}\right)$, it transformed from the glassy state to high-elastic state, and became unstable. Thus the thermal property of the polystyrene microspheres could be used for temperature sensing below $80{ }^{\circ} \mathrm{C}$.

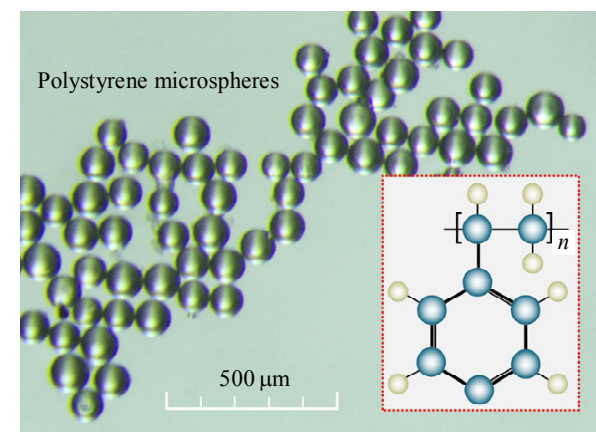

(a)

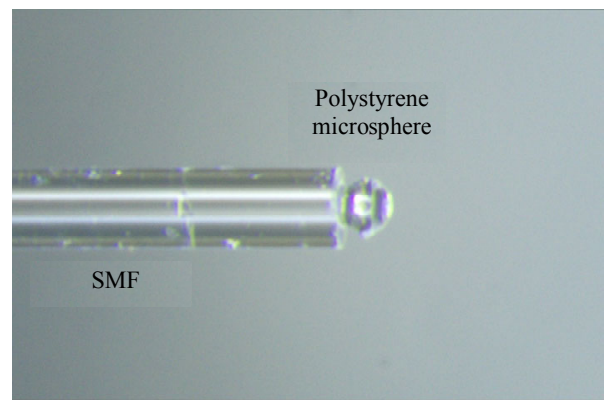

(b)

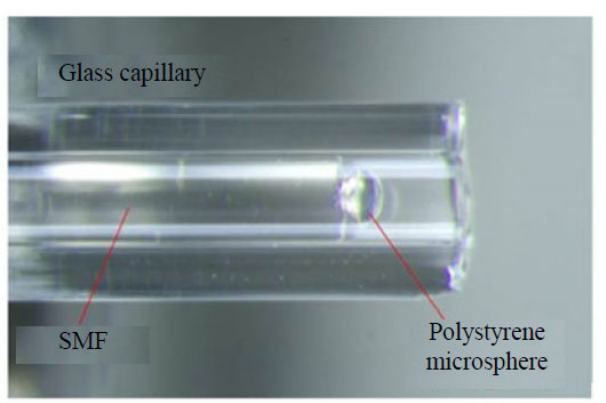

(c)

Fig. 3 Microscopic photographs of (a) the polystyrene microsphere sample, (b) a fiber tip attached with a polystyrene microsphere, and (c) the fiber tip polystyrene microsphere sensor with a glass capillary.

The fabricated fiber tip polystyrene microsphere sensors with and without a glass capillary are shown in Figs.3(b) and 3(c), respectively. The optical fiber used is the standard SMF G652D fabricated by Corning Inc., which has a core diameter of $9 \mu \mathrm{m}$, and a cladding diameter of $125 \mu \mathrm{m}$. The glass capillary 
has an inner diameter of $128 \mu \mathrm{m}$ and an outer diameter of $420 \mu \mathrm{m}$.

Fabrication repeatability of the sensor mainly depends on two factors, the consistency of the diameter of the polystyrene microspheres and the dislocation of the microsphere fixed in the center of the fiber facet. If diameters of the monodisperse polymer microsphere distribute in a relatively large range, the sensitivities of the fabricated sensors will be relatively different from each other. To reduce response differences between sensors, better monodispersed polystyrene microspheres with much smaller standard deviation of diameters can be used.

In the fabrication process, when gluing the polystyrene microsphere to the center of the fiber tip (Step 3), special attention should be paid to its transverse position. Since the fiber core is only $9 \mu \mathrm{m}$, dislocation of the microsphere from the tip center may reduce the reflected optical power, or even no spectral signal can be received. To minimize the dislocation, the following method can be used. Illuminate the fiber tip by a broadband source, at the same time, continuously monitor its reflection spectrum with an optical spectrum analyzer (OSA). When the polystyrene microsphere is very close to the tip center, there will appear a spectrum with multiple peaks. With a fixed distance between the fiber tip and the microsphere, the transverse position of the microsphere is finely tuned through a multi-axis translation stage. Only when the center of microsphere is at the axis of the fiber core, we can get a maximum signal on the OSA. By the optimization of the reflected spectral signal, the polystyrene microsphere can be optimally fixed to the fiber tip center.

\section{Temperature sensing mechanism}

When a light is coupled into the fiber tip polystyrene microsphere sensor, two reflected lights will be formed and returned back to the lead-in fiber. One is returned back from the interface between the fiber facet and the rear surface of microsphere, and the other is returned from the front surface of microsphere. The two reflected lights interfere with each other.

Since the reflectivities of the two interfaces are relatively low, according to the multi-beam interferencing theory, the total intensity of the interferencing reflected lights can be given by

$$
I=I_{1}+I_{2}+2 \sqrt{I_{1} I_{2}} \cos \phi
$$

where $I_{1}$ and $I_{2}$ are the intensities of the light reflected from the front and rear interfaces of the polystyrene microsphere, respectively. And the phase factor $\phi$ can be given by

$$
\phi=\frac{4 \pi}{\lambda} O P D+\phi_{0}
$$

where $\phi_{0}$ is an initial phase constant, $O P D=n d$ ( $n$ is the refractive index of the polystyrene, $d$ is the diameter of the polystyrene microsphere, i.e., the FP cavity length), and $\lambda$ is the optical wavelength.

As external temperature changes, since the diameter and refractive index of the polystyrene microsphere will change accordingly, because of the thermal expansion and thermo-optic effects, the OPD between the two reflected light will linearly vary with the temperature changing, which leads to the phase shift of the interferometric signal. By the monitoring of the phase shift, the external temperature change can be extracted.

\section{Experiment and analysis}

An experimental setup for the characterization of the fiber tip polystyrene microsphere sensor was built as shown in Fig. 4, which is composed of a super luminescent diode (SLD) [S5FC1550S-A2, Thorlabs Inc., central wavelength $1568 \mathrm{~nm}, 3-\mathrm{dB}$ bandwidth $90 \mathrm{~nm}$, maximum output power $3 \mathrm{~mW}$, of which, the output spectrum is shown in Fig.5(a)] an optical circulator, an OSA (MS9740A, Anritsu Inc., wavelength range of $0.6 \mu \mathrm{m}-1.75 \mu \mathrm{m}$, best wavelength resolution of $0.03 \mathrm{~nm}$ ), and an computer. The microsphere sensor was inserted into a high temperature furnace for temperature control, which had a temperature resolution of $1^{\circ} \mathrm{C}$. The wideband light output from the SLD illuminated the FP microsphere sensor through the optical circulator. 
The reflected light containing the temperature modulated information of the microsphere was returned back to the optical circulator and transmitted into the OSA to get the reflection spectrum, which was analyzed through a computer to interrogate the temperature.

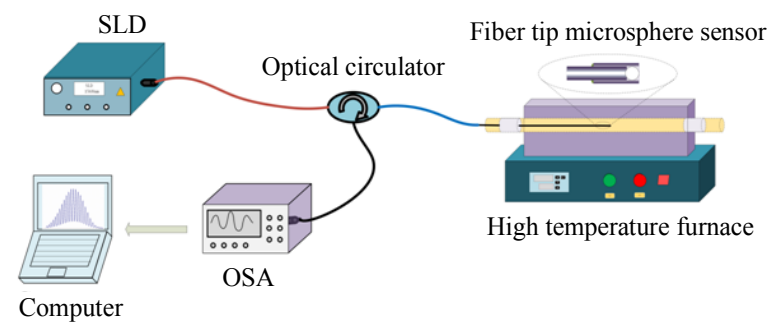

Fig. 4 Experimental setup for the investigation of the fiber tip polystyrene microsphere temperature sensor.

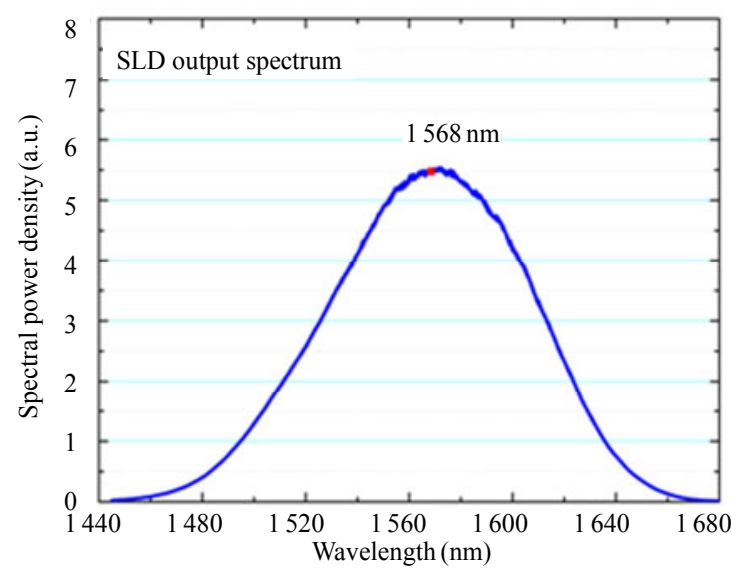

(a)
The reflection spectrum of the fiber tip polystyrene microsphere temperature sensor is similar to that shown in Fig. 5(a), which was measured for a sphere with an initial diameter of $91.706 \mu \mathrm{m}$ at a temperature of $20{ }^{\circ} \mathrm{C}$, and was a typical reflection spectrum of an FP cavity. Since the cavity length is relatively short, only limited several peaks appeared in the spectral range of $1472 \mathrm{~nm}-$ $1672 \mathrm{~nm}$. With an increase in the temperature, wavelengths corresponding to the reflection dips or peaks blueshifted to the shorter wavelength direction, which was caused by the shrinking of the polystyrene microsphere with the increasing in the temperature.

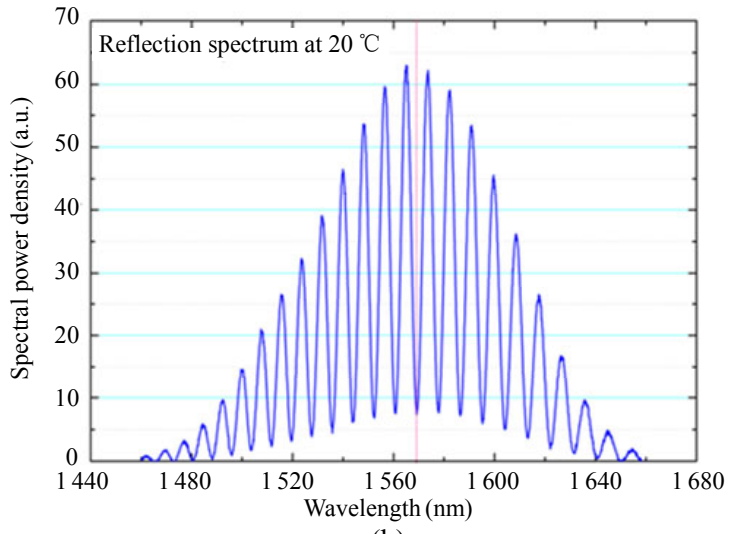

(b)

Fig. 5 Optical spectra of the SLD source and the sensor: (a) output spectrum of the SLD and (b) full reflection spectrum of the fiber-tip microsphere sensor with an initial diameter of $91.706 \mu \mathrm{m}$ at a temperature of $20{ }^{\circ} \mathrm{C}$.

In Fig. 6(a), the wavelength shift of the the reflection dip marked in Fig. 5(b) is given for a temperature range of $20{ }^{\circ} \mathrm{C}-70{ }^{\circ} \mathrm{C}$ with a temperature step of $10{ }^{\circ} \mathrm{C}$. We also give out the relationship between the dip wavelength and the temperature in Fig. 6(b), which is with a good linear manner, and the wavelength-temperature sensitivity can be found to be $-0.0065 \mathrm{~nm} /{ }^{\circ} \mathrm{C}$. The sensitivity is several times of the result in [18], but lower than that in [7].

The OPD caused by the polystyrene microsphere can be calculated through the reflection spectrum by the peak-to-peak method. The temperature response of the OPD is shown in Fig. 7. It can be found that, the relationship between the OPD and the temperature has a good linearity during a complete heating and cooling process. Although there exists some small deviations between the heating and cooling processes, it can still be safely concluded that the repeatability is good. However, the temperature should not reach or exceed the glass transition temperature $80{ }^{\circ} \mathrm{C}$, otherwise, the repeatability cannot be guaranteed. Through the linear fitting, the OPD-temperature sensitivity is $-0.61796 \mathrm{~nm} /{ }^{\circ} \mathrm{C}$, and the $R^{2}$ coefficient is 0.9916 . The experiment shows that the fiber tip polystyrene microsphere temperature sensor has a high temperature sensitivity and good linearity. 


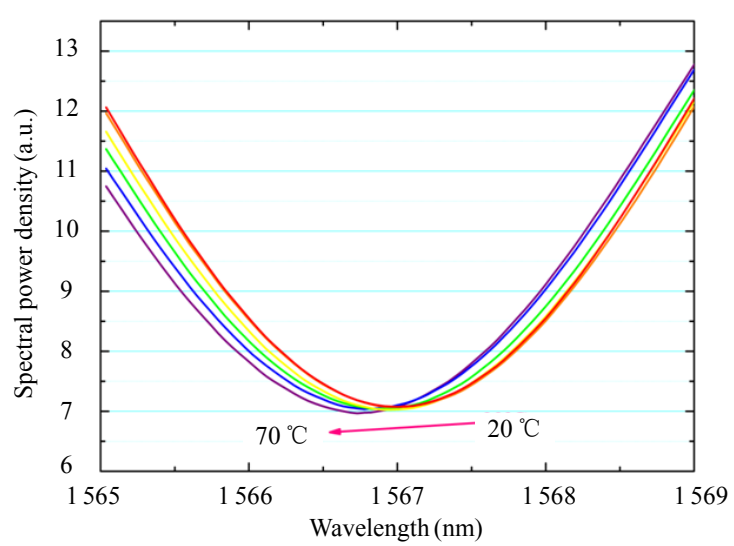

(a)

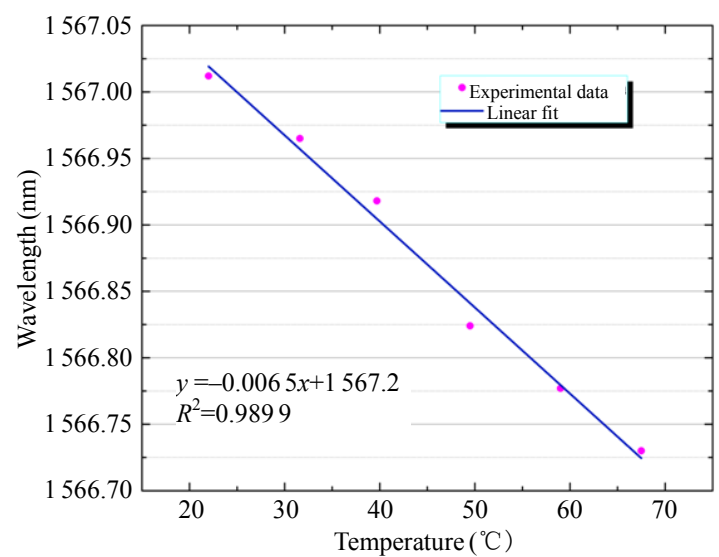

(b)

Fig. 6 Wavelength shift caused by temperature change: (a) wavelength shift of one reflection dip in a temperature range of $20{ }^{\circ} \mathrm{C}-70{ }^{\circ} \mathrm{C}$ and (b) relationship between the dip wavelength and the temperature.

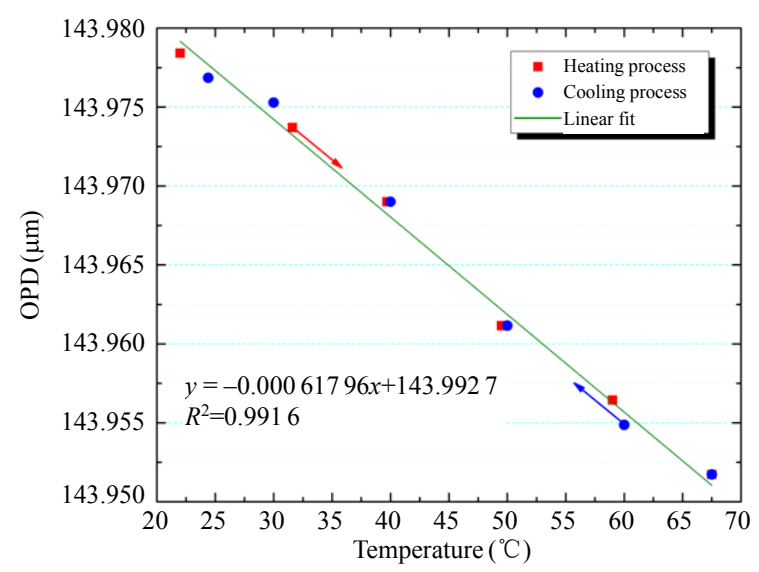

Fig. 7 Relationship between OPD and the temperature.

\section{Conclusions}

In summary, we proposed and fabricated a miniature fiber-optic temperature sensor by the gluing of a polystyrene microsphere on the tip center of a vertically cut single mode fiber. By using a polystyrene microsphere with a diameter of about $91.7 \mu \mathrm{m}$, a high OPD-temperature sensitivity of $-0.61796 \mathrm{~nm} /{ }^{\circ} \mathrm{C}$ in a temperature range of $20{ }^{\circ} \mathrm{C}-70{ }^{\circ} \mathrm{C}$ was achieved. The sensor also showed a good linearity of 0.9916 . The sensor structure is simple, compact, and easy to fabricate, and can be used in various applications.

\section{Acknowledgment}

This research was partially funded by Natural Science Basic Research Project of Shaanxi Province, China (Grant No. 2020JM-560), Key Scientific Research Plan of Education Department of Shaanxi Province, China (Grant No. 20JY028), and the National Natural Science Foundation of China (Grant No. 61704134).

Open Access This article is distributed under the terms of the Creative Commons Attribution 4.0 International License (http://creativecommons.org/licenses/by/4.0/), which permits unrestricted use, distribution, and reproduction in any medium, provided you give appropriate credit to the original author(s) and the source, provide a link to the Creative Commons license, and indicate if changes were made.

\section{References}

[1] K. S. Kim, J. W. Yoo, S. K. Kim, and B. Y. Kim, "Embedded intrinsic Fabry-Perot optical fiber sensors in the cement concrete structure for health monitoring system," SPIE, 1996, 2718: 218-231.

[2] B. Qi, G. R. Pickrell, P. Zhang, Y. Duan, W. Peng, J. C. Xu, et al., "Fiber optic pressure and temperature sensors for oil down hole application," Fiber Optic Sensor Technology \& Applications. International Society for Optics and Photonics, 2002, 4578: 182190.

[3] K. A. Murphy, M. F. Gunther, A. M. Vengsarkar, and R. O. Claus, "Fabry-Perot fiber-optic sensors in fullscale fatigue testing on an F-15 aircraft," Applied Optics, 1992, 31(4): 431-433.

[4] G. Minas, J. C. Ribeiro, J. S. Martins, R. F. Wolffenbuttel, and J. H. Correia, "An array of FabryPerot optical-channels for biological fluids analysis," Sensors \& Actuators A Physical, 2004, 115(2-3): 362-367.

[5] M. Jedrzejewska-Szczerska, "Response of a new low-coherence Fabry-Perot sensor to Hematocrit 
levels in human blood," Sensors, 2014, 14(4): 6965-6976.

[6] J. Ma, W. Jin, H. L. Ho, and J. Y. Dai, "Highsensitivity fiber-tip pressure sensor with graphene diaphragm," Optics Letters, 2012, 37(13): 24932495.

[7] D. Donlagic and E. Cibula, "All-fiber highsensitivity pressure sensor with $\mathrm{SiO}_{2}$ diaphragm," Optics Letters, 2005, 30(16): 2071-2073.

[8] F. Guo, T. Fink, M. Han, L. Koester, J. Turner, and J. Huang, "High-sensitivity, high-frequency extrinsic Fabry- Perot interferometric fiber-tip sensor based on a thin silver diaphragm," Optics Letters, 2012, 37(9): 1505-1507.

[9] Z. Zhang, C. Liao, J. Tang, Z. Bai, K. Guo, M. Hou, et al., "High-sensitivity gas-pressure sensor based on fiber-tip PVC diaphragm Fabry-Pérot interferometer," Journal of Lightwave Technology, 2017, 35(18): 4067-4071.

[10] X. Qi, S. Wang, J. Jiang, K. Liu, X. Wang, Y. Yang, et al., "Fiber optic Fabry-Perot pressure sensor with embedded MEMS micro-cavity for ultra-high pressure detection," Journal of Lightwave Technology, 2019, 37(11): 2719-2725.

[11] M. Li, M. Wang, and H. Li, "Optical MEMS pressure sensor based on Fabry-Perot interferometry," Optics Express, 2006, 14(4): 1497-1504.

[12] Y. Zhang, L. Yuan, X. Lan, A. Kaur, J. Huang, and H. Xiao, "High-temperature fiber-optic Fabry-Perot interferometric pressure sensor fabricated by femtosecond laser," Optics Letters, 2013, 38(22): 4609-4612.

[13] J. Liu, L. Yuan, J. Lei, W. Zhu, B Cheng, Q. Zhang, et al., "Micro-cantilever-based fiber optic hydrophone fabricated by a femtosecond laser," Optics Letters,
2017, 42(13): 2459-2462.

[14] C. Liao, S. Liu, L. Xu, C. Wang, Y. Wang, Z. Li, et al., "Sub-micron silica diaphragm-based fiber-tip Fabry-Perot interferometer for pressure measurement," Optics Letters, 2014, 39(10): 2827-2830.

[15] J. Ma, J. Ju, L. Jin, and W. Jin, "A compact fiber-yip micro-cavity sensor for high-pressure measurement," IEEE Photonics Technology Letters, 2011, 23(21): 1561-1563.

[16] J. Ma, W. Jin, and L. Jin, "A novel fiber-tip microcavity sensor for high temperature application," in 21st International Conference on Optical Fiber Sensors, Ottawa, 2011, 7753: 775312.

[17] W. Chen, D. Wang, B. Xu, Z. Wang, and C. Zhao, "Fabry-Perot interferometer fiber tip sensor based on a glass microsphere glued at the etched end of multimode fiber," Optical Engineering, 2017, 56(5): 057107.

[18] X. Jiang, D. Chen, J. Shao, G. Feng, and J. Yang, "Low-cost fiber-tip Fabry-Perot interferometer and its application for high temperature sensing," Chinese Optics Letters, 2014, 12(s1): S10609.

[19] X. Tan, X. Li, Y. Geng, Z. Yin, L. Wang, W. Wang, et al., "Polymer microbubble-based Fabry-Perot fiber interferometer and sensing applications," IEEE Photonics Technology Letters, 2015, 27(19): 20352038.

[20] D. Duan, Y. Rao, Y. S. Hou, and T. Zhu, "Microbubble based fiber-optic Fabry-Perot interferometer formed by fusion splicing single-mode fibers for strain measurement," Applied Optics, 2012, 51(8): 10331036.

[21] J. Song and M. Winnik, "Cross-linked, monodisperse, micron-sized polystyrene particles by two-stage dispersion polymerization," Macromolecules, 2005, 38(20): 8300-8307. 